\title{
Non-monotonic Keplerian velocity profiles around near-extreme braneworld Kerr black holes
}

\author{
Zdeněk Stuchlík, Martin Blaschke and Petr Slaný \\ Institute of Physics, Faculty of Philosophy and Science, Silesian university in \\ Opava, Bezručovo nám. 13, CZ-746 01 Opava, Czech Republic \\ E-mail: MartinBlaschke@seznam.cz, Petr.Slany@fpf.slu.cz, \\ Zdenek.Stuchlik@fpf.slu.cz
}

\begin{abstract}
We study the non-monotonic Keplerian velocity profiles related to locally non-rotating frames (LNRF) in the field of near-extreme braneworld Kerr black holes and naked singularities in which the non-local gravitational effects of the bulk are represented by a braneworld tidal charge $b$ and the $4 \mathrm{D}$ geometry of the spacetime structure is governed by the Kerr-Newman geometry. We show that positive tidal charge has a tendency to restrict the values of the black hole dimensionless spin $a$ admitting existence of the non-monotonic Keplerian LNRFvelocity profiles; the non-monotonic profiles exist in the black hole spacetimes with tidal charge smaller than $b=0.41005$ (and spin larger than $a=0.76808$ ). With decreasing value of the tidal charge (which need not be only positive), both the region of spin allowing the non-monotonicity in the LNRF-velocity profile around braneworld Kerr black hole and the velocity difference in the minimummaximum parts of the velocity profile increase implying growing astrophysical relevance of this phenomenon.
\end{abstract}

PACS numbers: 04.70.-s, 04.20.-q, 98.80.-k, 04.50.-h

Submitted to: Class. Quantum Grav. 
Non-monotonic Keplerian velocity profiles around brany Kerr black holes

\section{Introduction}

Fast rotating black holes play a crucial role in understanding processes observed in quasars and Active Galactic Nuclei (AGN) or in microquasars. It has been shown that supermassive black holes in AGN evolve into states with dimensionless spin $a \sim 1$ due to accretion from thin discs $[1,2]$. This statement is supported by analysis of profiled X-ray (Fe56) lines observed in some AGN (e.g. in MCG-6-30-15) [3, 4, 5] and in some microquasars (e.g., GRS 1915+105) [6]. Evidence for the existence of near-extreme Kerr black holes comes from high-frequency quasi-periodic oscillations (QPOs) of observed X-ray flux in some microquasars [7, 8]. A fast rotating black hole could be also located in the Galaxy center source Sqr A* $[9,10,11]$.

It is widely accepted that the phenomena observed in AGN and microquasars are related to accretion discs orbiting Kerr black holes. However, we can consider also the possibility to explain these phenomena by Kerr superspinars with external field described by the geometry of Kerr naked singularity spacetime [12]. Then both accretion and related optical effects and the QPOs effects enable us to find clear signature of the Kerr superspinar presence [13, 14, 15, 16, 17, 18].

Properties of accretion discs can be appropriately represented by circular orbits of test particles or fluid elements orbiting black holes (superspinars). The local properties can be efficiently expressed when related to the locally non-rotating frames (LNRF), since these frames corotate with the spacetime in a way that enables to cancel the frame-dragging effects as much as possible [19]. A new phenomenon related to the LNRF-velocity profiles of matter orbiting near-extreme Kerr black holes has been found by B. Aschenbach [9, 20, 21], namely a non-monotonicity in the velocity profile of the Keplerian motion in the field of Kerr black holes with dimensionless spin $a>0.9953$. Such a hump in the LNRF-velocity profile of the corotating orbits is a typical and relatively strong feature in the case of Keplerian motion in the field of Kerr naked singularities, but in the case of Kerr black holes it is a very small effect appearing for near-extreme black holes only - see Figure 1. In the naked singularity case we call the orbits to be of 1st family rather than corotating, since these can be retrograde relative to the LNRF in vicinity of the ring singularity for small values of spin $(a<5 / 3)$, while they are corotating for larger values of spin [15]; the humpy character of the LNRF-velocity profile ceases for naked singularities with $a>4.0014-$ as demonstrated in the Figure 1. A study of non-Keplerian distribution of specific angular momentum $(l=$ const $)$, related to geometrically thick discs of perfect fluid, has shown that the "humpy" LNRF-velocity profile appears for near-extreme Kerr black holes with $a>0.9998$ [21]. The humpy LNRF-velocity profile emerges in the ergosphere of near-extreme Kerr black holes, at vicinity of the marginally stable circular orbit. Maximal velocity difference between the local minimum and maximum of the humpy Keplerian velocity profiles is $\Delta v \approx 0.07 c$ and takes place for $a=1$ [22].

Using idea of "hump-induced" oscillations related to humpy LNRF-velocity profiles, the Extended Orbital Resonance Model (EXORM) was developed and applied to explain complex QPO patterns observed in some black hole sources [22, 23, 24]. In the EXORM, the resonant phenomena between oscillations with orbital frequencies (Keplerian and epicyclic - radial or vertical) and so called "humpy" frequency, given by the maximal slope of the LNRF-velocity profile in its humpy part, are assumed to appear at the radius where the "humpy" oscillations are expected to be generated by the non-monotonicity of the LNRF-velocity profile [23]. The EXORM is able to explain all five high-frequency QPOs observed in the microquasar GRS 1915+105 

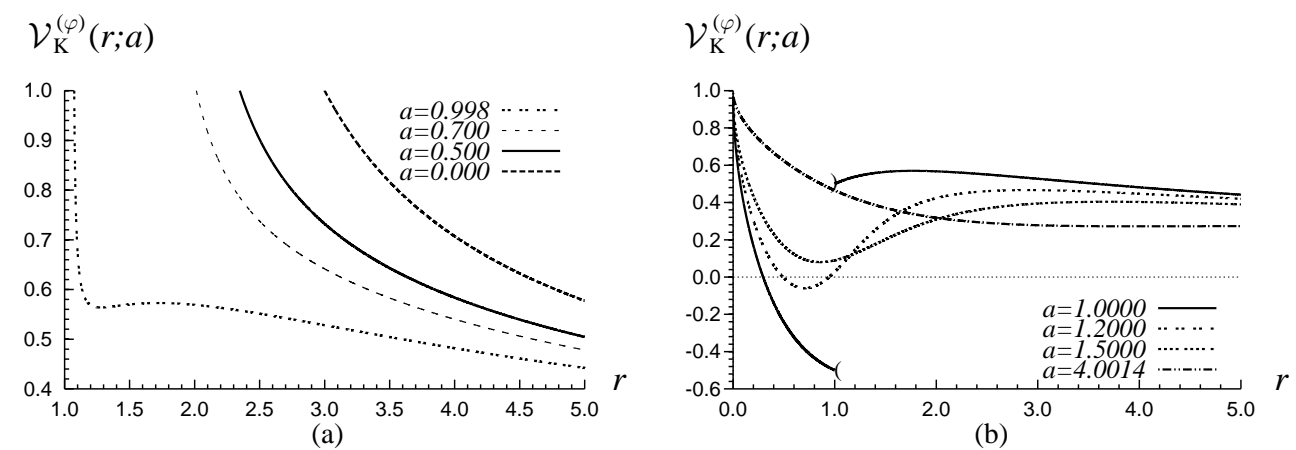

Figure 1. Keplerian velocity profiles related to the LNRF.

(a): Kerr black holes - the velocity profiles presented for some values of the black hole spin. The Aschenbach effect appears for near-extreme black holes and is weak.

(b): Kerr naked singularities - the velocity profiles are given for some values of the spin, demonstrating existence of Aschenbach effect for orbits with negative valued velocity. For completeness, the velocity profile is given also for extreme black hole, demonstrating velocity jump at $r=1$.

by the humpy frequency, the radial (and vertical) epicyclic frequencies and their simple combinations taken at the common "humpy" radius, implying the black hole parameters of the source $M=14.8 M_{\odot}, \mathrm{a}=0.9998$ [22], in good agreement with estimates given by different methods [6,20]. This model can give interesting results also in the case of the X-ray binary system XTE J1650-500 [24], and an ULX candidate system NGC 5408 X-1. On the other hand, QPOs observed in Sqr A* [9] cannot be explained by the EXORM [25].

The humpy LNRF-velocity profiles for both Keplerian and $l=$ const specific angular momentum distributions of orbiting matter were studied in the Kerr-de Sitter and Kerr-anti-de Sitter black hole spacetimes and values of the black hole spin $a$ allowing for existence of the humpy profiles were found in dependence on the value of the cosmological constant $[26,27]$.

Last decade gave rise to a plenty of models modifying the 4D Einstein general relativity due to hidden dimensions, therefore, it is interesting to investigate the Aschenbach effect in rotating black hole (naked singularity) spacetimes allowed in alternative gravitational theories. The string theories, describing gravity as higherdimensional interaction appearing to be effectively $4 \mathrm{D}$ at low energies, inspired braneworld models assuming the observable universe to be a 3-brane, i.e. "domain wall", to which the non-gravitational matter fields are confined, while gravity enters the extra spatial dimensions that could be much larger than $l_{p} \sim 10^{-33} \mathrm{~cm}$. The model of Randall and Sundrum (RS model) [28] allows gravity localized near the brane with an infinite size extra dimension while the warped spacetime satisfies the 5D Einstein equations with negative cosmological constant. An arbitrary energy-momentum tensor could then be allowed on the brane and effective 4D Einstein equations have to be satisfied on the brane. The RS model implies standard 4D Einstein equations in the low energy limit, but significant deviations occur at high energies, near black holes or compact stars. The combination of high-energy (local) and bulk stress (non-local) effects alters the matching problem on the brane in comparison with the standard $4 \mathrm{D}$ gravity [29]. The bulk gravity stresses imply that the matching conditions do not 
have unique solution on the brane and the $5 \mathrm{D}$ Weyl tensor is needed as a minimum condition for uniqueness.

No exact solution of the 5D braneworld Einstein equations is known at present, but a numerical solution has been found quite recently [30]. On the other hand, $4 \mathrm{D}$ stationary and axisymmetric vacuum solution describing a braneworld rotating black hole has been found by solving the braneworld constrained equations under an assumption of specialized form of the metric (namely of the Kerr-Schild form) [31]. Of course, it is not an exact solution satisfying the full system of 5D equations, but in the framework of the constrained equations it represents a consistent rotating black hole solution reflecting the influence of the extra dimension through a single braneworld parameter. The braneworld rotating black holes are described by the metric tensor of the Kerr-Newman form with the braneworld tidal charge $b$ determining the 5D non-local gravitational coupling between the brane and the bulk [31]. For non-rotating braneworld black holes, the metric is reduced to the ReissnerNordström form containing the tidal charge [32]. This spacetime can also represent the external field of braneworld neutron stars described by the uniform density internal spacetime [29]. Influence of the braneworld tidal charge on physical processes has been extensively investigated for both the black holes [33, 34, 35, 36, 37, 38] and neutron stars [39, 40, 41, 42, 43], or in the weak field limit [44, 45]. In the case of microscopic black holes, an experimental evidence is assumed in LHC [46].

The braneworld tidal charge can be, in principle, both positive and negative, but the negative values are probably more relevant [32]. Notice that for $b>0$ the braneworld spacetime can be identified with the Kerr-Newman spacetime by $b \rightarrow Q^{2}$, where $Q^{2}$ is the squared electric charge; however it is not the KerrNewman background since the electromagnetic part of this background is missing. Some astrophysically relevant restrictions on the value of the tidal charge $b$ were obtained both in the weak-field limit [44] and in the strong-field limit [39].

Here, we shall study existence of the humpy LNRF-velocity profiles in the field of braneworld rotating black holes considering both negative and positive values of the braneworld tidal charge. Our results related to $b>0$ are relevant also in the case of the standard Kerr-Newman spacetimes (with $b \rightarrow Q^{2}$ ), for uncharged particles. We restrict our attention to the Keplerian LNRF-velocity profiles postponing the study of perfect fluid configurations to future work.

\section{Effective gravitational equations in braneworld models}

In the 5D warped space models of Randall and Sundrum, the gravitational field equations in the bulk can be expressed in the form [32, 47]

$$
\tilde{G}_{A B}=\tilde{k}^{2}\left[-\tilde{\Lambda} \tilde{g}_{A B}+\delta(\chi)\left(-\lambda g_{A B}+T_{A B}\right)\right]
$$

where the fundamental $5 \mathrm{D}$ Planck mass $\tilde{M}_{\mathrm{P}}$ enters via $\tilde{k}^{2}=8 \pi / \tilde{M}_{\mathrm{P}}^{3}, \lambda$ is the brane tension, and $\tilde{\Lambda}$ is the negative bulk cosmological constant; $g_{A B}=\tilde{g}_{A B}-n_{A} n_{B}$ is the induced metric on the brane, with $n_{A}$ being the unit vector normal to the brane.

The effective gravitational field equations induced on the brane are determined by the bulk field equations (1), the Gauss-Codazzi equations and the generalised matching Israel conditions. They can be expressed in the form of modified Einstein's equations containing additional terms reflecting bulk effects onto the brane [32, 47]

$$
G_{\mu \nu}=-\Lambda g_{\mu \nu}+k^{2} T_{\mu \nu}+\tilde{k}^{2} S_{\mu \nu}-\mathcal{E}_{\mu \nu}
$$




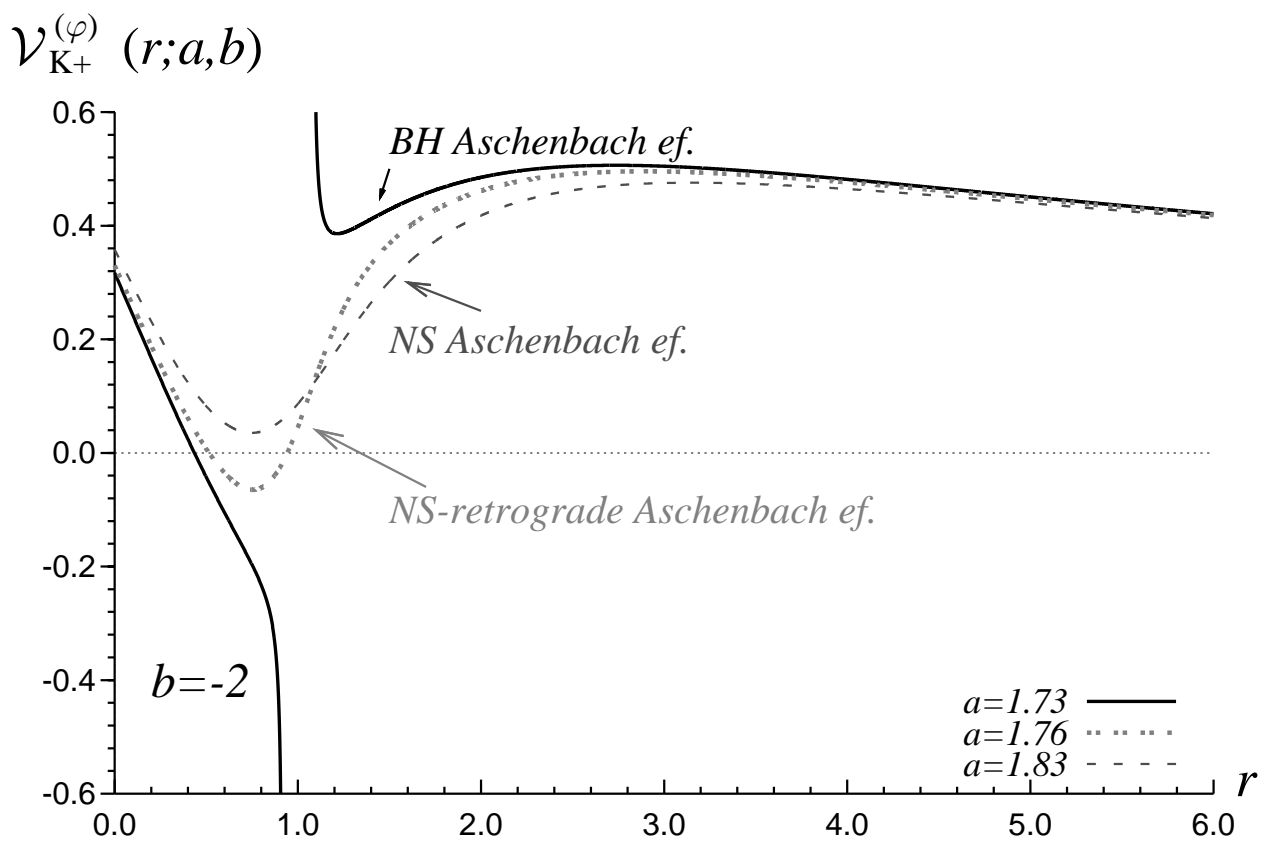

Figure 2. Non-monotonic plus-family orbital velocity profiles in braneworld Kerr black hole and naked singularity spacetimes with the tidal charge $b=-2$, given for three appropriately chosen values of spin $a$ reflecting the whole variety of possible behaviour related to the Aschenbach effect.

where $k^{2}=8 \pi / M_{\mathrm{P}}^{2}$, with $M_{\mathrm{P}}$ being the braneworld Planck mass. The relations of the energy scales and cosmological constants are given in the form

$$
M_{\mathrm{P}}=\sqrt{\frac{3}{4 \pi}}\left(\frac{\tilde{M}_{\mathrm{P}}^{2}}{\sqrt{\lambda}}\right) \tilde{M}_{\mathrm{P}} ; \quad \Lambda=\frac{4 \pi}{\tilde{M}_{\mathrm{P}}^{3}}\left[\tilde{\Lambda}+\left(\frac{4 \pi}{3 \tilde{M}_{\mathrm{P}}^{3}}\right) \lambda^{2}\right], .
$$

Local bulk effects on the matter are determined by the "squared energy-momentum" tensor $S_{\mu \nu}$, while the non-local bulk effects are given by the tensor $\mathcal{E}_{\mu \nu}$.

Assuming zero cosmological constant on the brane $(\Lambda=0)$ we arrive to the condition

$$
\tilde{\Lambda}=-\frac{4 \pi \lambda^{2}}{3 \tilde{M}_{\mathrm{P}}^{3}} .
$$

In the vacuum case, $T_{\mu \nu}=0=S_{\mu \nu}$, the effective gravitational field equations on the brane reduce to the form [47]

$$
R_{\mu \nu}=-\mathcal{E}_{\mu \nu}, \quad R_{\mu}{ }^{\mu}=0=\mathcal{E}_{\mu}{ }^{\mu}
$$

implying divergence constraint [47]

$$
\nabla^{\mu} \mathcal{E}_{\mu \nu}=0
$$

where $\nabla_{\mu}$ denotes the covariant derivative on the brane. 


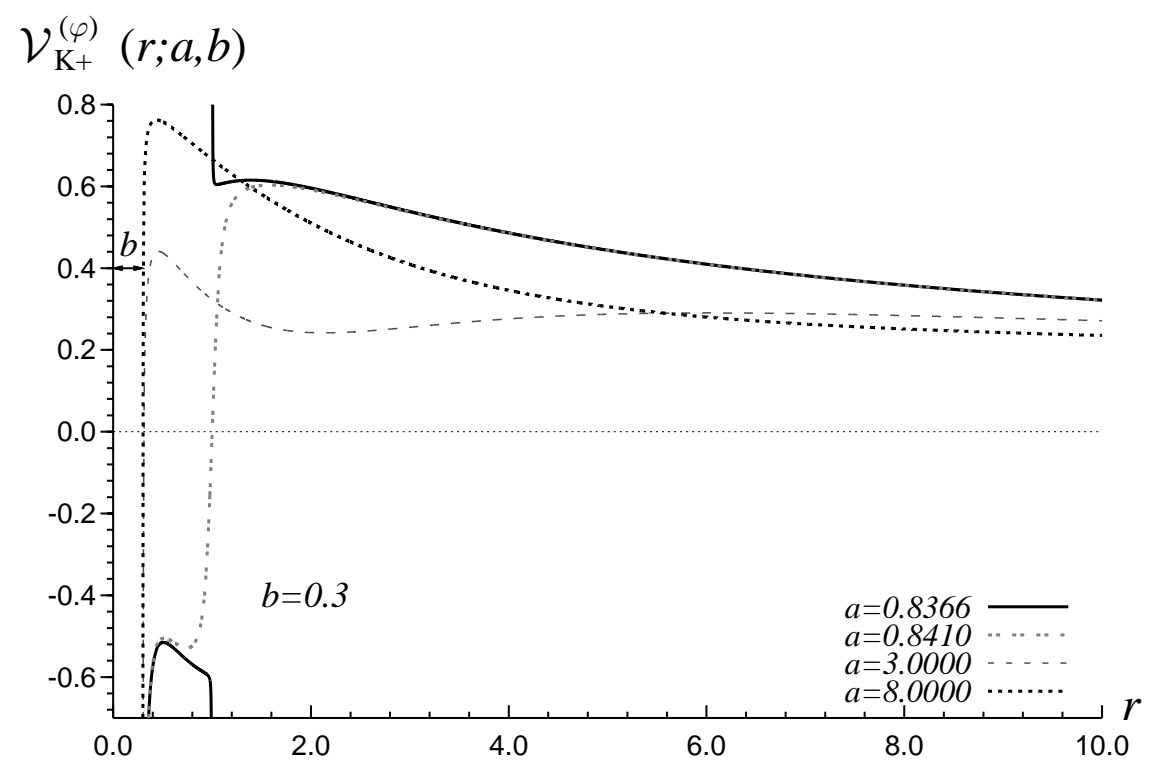

Figure 3. Non-monotonic plus-family orbital velocity profiles in braneworld Kerr black hole and naked singularity spacetimes with the tidal charge $b=0.3$.

The equation (6) represents Bianchi identities on the brane, i.e., an integrability condition for the field equations $R_{\mu \nu}=-\mathcal{E}_{\mu \nu}$ [31]. For stationary and axisymmetric (or static, spherically symmetric) solutions, (5) and (6) form a closed system of equations on the brane.

The $4 \mathrm{D}$ general relativity energy-momentum tensor $T_{\mu \nu}\left(\right.$ with $\left.T_{\mu}{ }^{\mu}=0\right)$ can be formally identified to the bulk Weyl term on the brane due to the correspondence

$$
k^{2} T_{\mu \nu} \quad \leftrightarrow \quad-\mathcal{E}_{\mu \nu} .
$$

The general relativity conservation law $\nabla^{\mu} T_{\mu \nu}=0$ then corresponds to the constraint equation on the brane (6). This behaviour indicates that the Einstein-Maxwell solutions in standard general relativity should correspond to constrained braneworld vacuum solutions. This was indeed shown in the case of braneworld (ReissnerNordström and Kerr-Newman) black hole solutions [31]. In both of these solutions the influence of the non-local gravitational effects of the bulk on the brane are represented by a single "braneworld" parameter $b$. The $1 / r^{2}$ behaviour of the second term in the Newtonian potential

$$
\Phi=-\frac{M}{M_{\mathrm{P}}^{2} r}+\frac{b}{2 r^{2}}
$$

inspired the name "tidal charge" for the parameter b [32].

\section{Orbital motion in the braneworld Kerr spacetimes}

Motion of test particles in the field of braneworld rotating black holes is given by the geodesic structure of the Kerr-Newman spacetimes with the tidal charge 


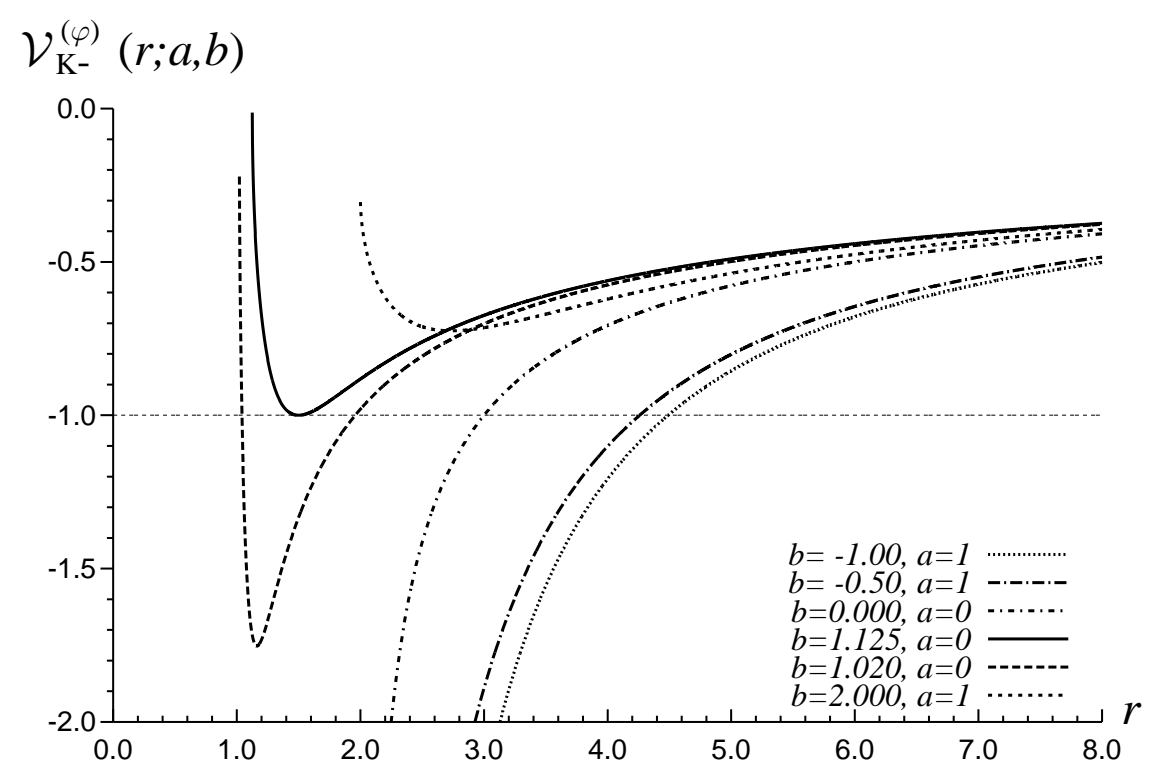

Figure 4. Minus-family orbital velocity profiles in braneworld Kerr black hole and naked singularity spacetimes.

b. The braneworld parameter reflects the tidal effects of the bulk space and has no influence on the motion of charged particles. The geodesic structure given by the Carter equations [48] is relevant for both uncharged and charged test particles. The circular test particle orbits of the braneworld Kerr black holes are identical to the circular geodesics of the Kerr-Newman spacetime with properly chosen charge parameter.

We shall study the Aschenbach effect, i.e., we look for the non-monotonicity (humps) in the LNRF-velocity profiles of Keplerian discs orbiting near-extreme braneworld Kerr black holes or naked singularities.

\subsection{Geometry}

Using standard Boyer-Lindquist coordinates $(t, r, \theta, \varphi)$ and geometric units $(c=G=$ 1 ), we can write the line element of rotating (Kerr) black hole on the 3D-brane in the form

$$
\begin{aligned}
& \mathrm{d} s^{2}=-\left(1-\frac{2 M r-b}{\Sigma}\right) \mathrm{d} t^{2}-\frac{2 a(2 M r-b)}{\Sigma} \sin ^{2} \theta \mathrm{d} t \mathrm{~d} \varphi+ \\
& +\frac{\Sigma}{\Delta} \mathrm{d} r^{2}+\Sigma \mathrm{d} \theta^{2}+\left(r^{2}+a^{2}+\frac{2 M r-b}{\Sigma} a^{2} \sin ^{2} \theta\right) \sin ^{2} \theta \mathrm{d} \varphi^{2},
\end{aligned}
$$

where

$$
\begin{aligned}
& \Delta=r^{2}-2 M r+a^{2}+b, \\
& \Sigma=r^{2}+a^{2} \cos ^{2} \theta,
\end{aligned}
$$


$a(r ; b)$

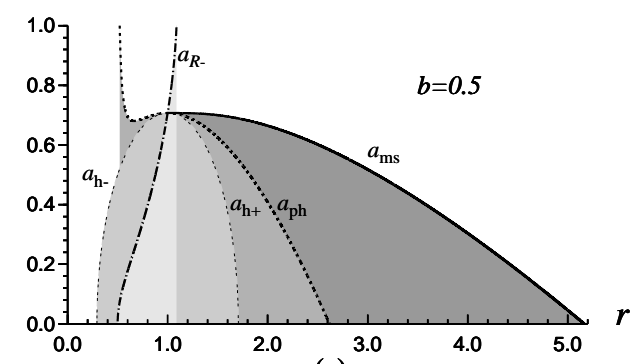

(a)

$$
a(r ; b)
$$

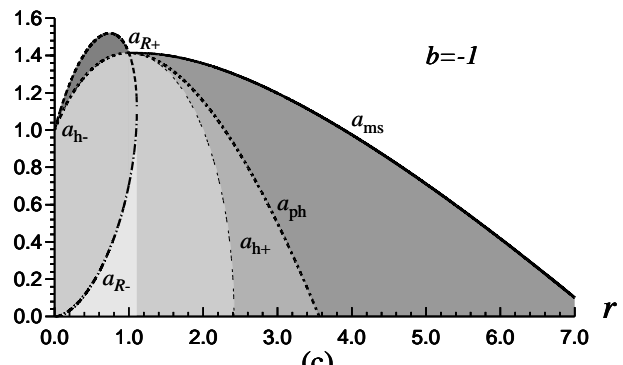

(c)

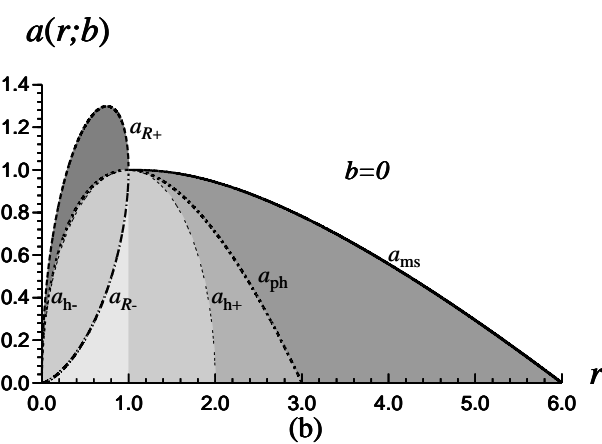

$a(r ; b)$

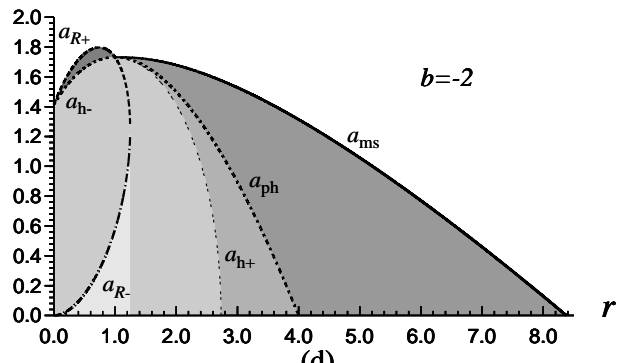

(d)

Figure 5. The loci of the photon circular orbits $a_{\mathrm{ph}}(r, b)$; the marginally stable orbits $a_{\mathrm{ms}}(r, b)$; function $a_{R \pm}(r, b)$ and function $a_{\mathrm{h} \pm}(r, b)$ defined implicitly by position of event horizon for appropriately chosen fixed values of $b$.

$M$ and $a=J / M$ are the mass parameter and the specific angular momentum of the background, while the braneworld parameter $b$, called "tidal charge", represents the imprint of non-local (tidal) gravitational effects of the bulk space [31]. The physical "ring" singularity of the braneworld rotating black holes (and naked singularities) is located at $r=0$ and $\theta=\pi / 2$, as in the Kerr spacetimes.

The form of the metric (9) is the same as that of the standard Kerr-Newman solution of the 4D Einstein-Maxwell equations, with tidal charge $b$ being replaced by squared electric charge $Q^{2}$ [49]. The stress tensor on the brane $\mathcal{E}_{\mu \nu}$ takes the form

$$
\begin{aligned}
\mathcal{E}_{t}^{t} & =-\mathcal{E}_{\varphi}^{\varphi}=-\frac{b}{\Sigma^{3}}\left[\Sigma-2\left(r^{2}+a^{2}\right)\right], \\
\mathcal{E}_{r}^{r} & =-\mathcal{E}_{\theta}^{\theta}=-\frac{b}{\Sigma^{2}}, \\
\mathcal{E}_{\varphi}^{t} & =-\left(r^{2}+a^{2}\right) \sin ^{2} \mathcal{E}_{t}^{\varphi}=-\frac{2 a b}{\Sigma^{3}}\left(r^{2}+a^{2}\right) \sin ^{2} \theta,
\end{aligned}
$$

that is fully analogical $\left(b \rightarrow Q^{2}\right)$ to components of the electromagnetic energymomentum tensor of the Kerr-Newmann solution in Einstein's general relativity [31]. For negative values of the tidal charge $(b<0)$, the values of the black hole spin $a>M$ are allowed. Such a situation is forbidden for the standard 4D Kerr black holes. In the following, we put $M=1$ in order to work with completely dimensionless formulae. 


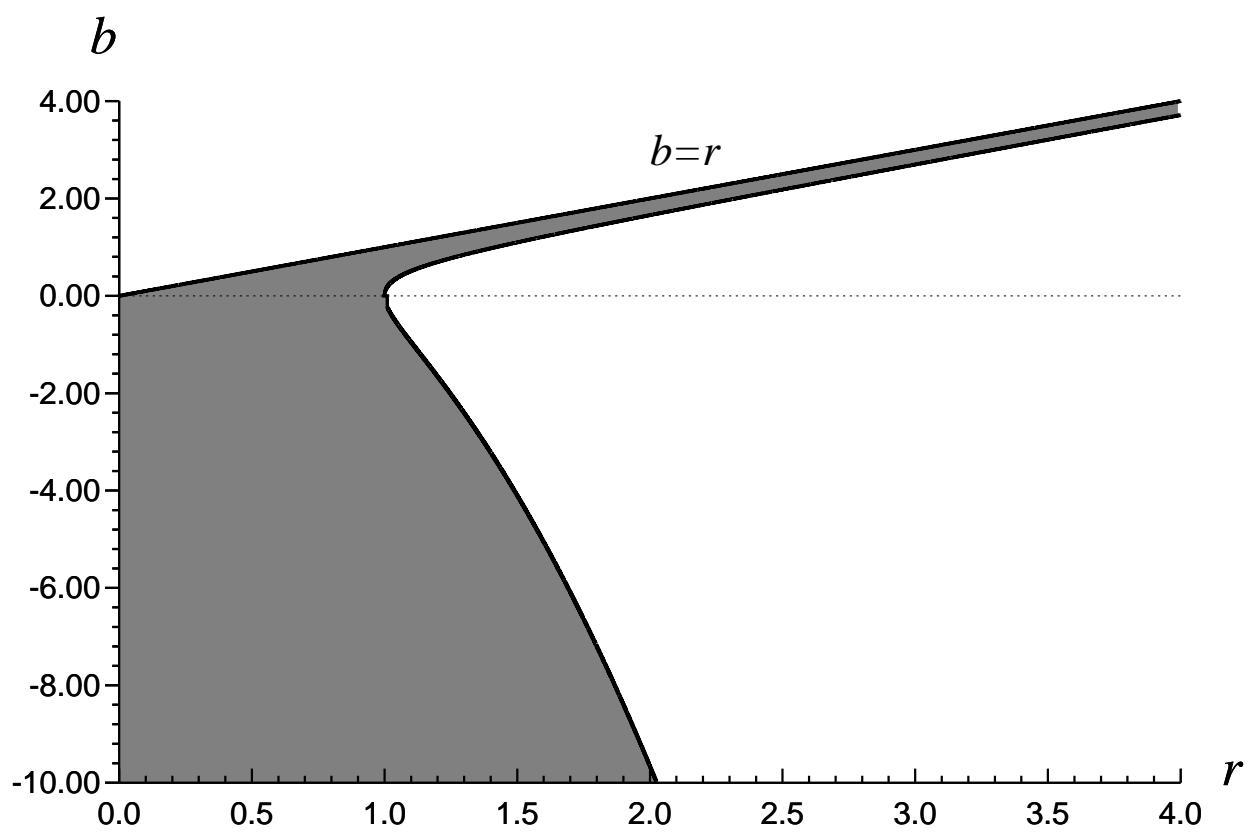

Figure 6. Naked singularity regions with potentially retrograde plus-family orbits.

\subsection{Locally non-rotating frames and orbital motion}

The orbital velocity of matter orbiting a braneworld Kerr black hole along circular orbits is given by appropriate projections of its 4 -velocity $U=\left(U^{t}, 0,0, U^{\varphi}\right)$ onto the tetrad of a locally non-rotating frame (LNRF) [19]

$$
\begin{aligned}
& \mathbf{e}^{(t)}=\left(\omega^{2} g_{\varphi \varphi}-g_{t t}\right)^{\frac{1}{2}} \mathbf{d} t, \\
& \mathbf{e}^{(\varphi)}=\left(g_{\varphi \varphi}\right)^{\frac{1}{2}}(\mathbf{d} \varphi-\omega \mathbf{d} t), \\
& \mathbf{e}^{(r)}=\left(\frac{\Sigma}{\Delta}\right)^{\frac{1}{2}} \mathbf{d} r, \\
& \mathbf{e}^{(\theta)}=\Sigma^{\frac{1}{2}} \mathbf{d} \theta,
\end{aligned}
$$

where $\omega$ is the angular velocity of the LNRF relative to distant observers and reads

$$
\omega=-\frac{g_{t \varphi}}{g_{\varphi \varphi}}=\frac{a(2 r-b)}{\Sigma\left(r^{2}+a^{2}\right)+(2 r-b) a^{2} \sin ^{2} \theta} .
$$

For the circular motion, the only non-zero component of the 3 -velocity measured locally in the LNRF is the azimuthal component that is given by

$$
\mathcal{V}_{\mathrm{LNRF}}^{(\varphi)}=\frac{[\Omega-\omega]}{\sqrt{\left(\omega^{2}-\frac{g_{t t}}{g_{\varphi \varphi}}\right)}}
$$




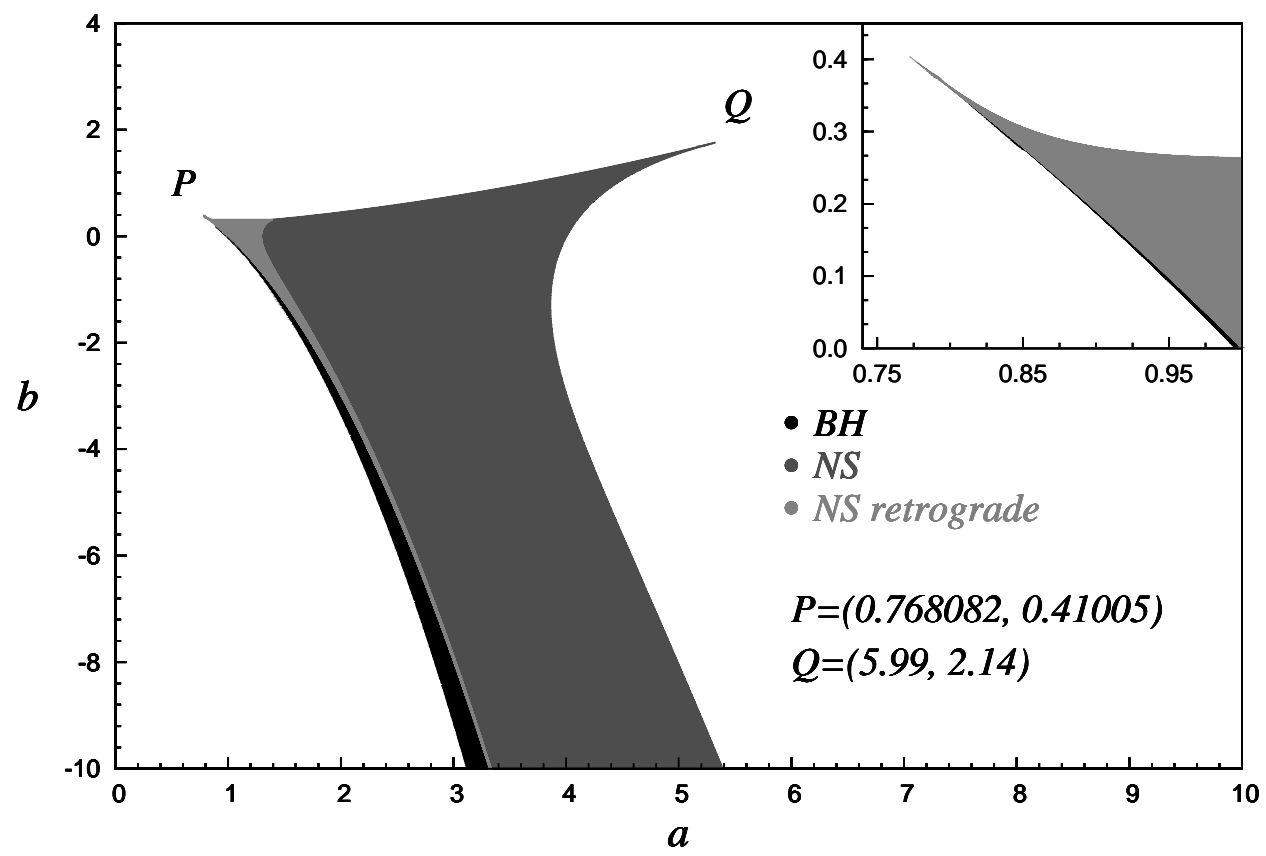

Figure 7. Classification of the braneworld Kerr spacetimes according to existence of the Aschenbach effect. The Aschenbach effect is allowed in the black region representing black holes, dark-grey region representing naked singularities with corotating orbits only, and lighter-grey region representing naked singularities with retrograde motion in the LNRF-velocity profile (corresponding to negative values of the function $\mathcal{V}_{\mathrm{K}}^{(\varphi)}$ ).

$$
=\frac{\left[\left(r^{2}+a^{2}\right)^{2}-a^{2} \Delta \sin ^{2} \theta\right] \sin \theta(\Omega-\omega)}{\Sigma \sqrt{\Delta}}
$$

where

$$
\Omega=\frac{U^{\varphi}}{U^{t}}=-\frac{l g_{t t}+g_{t \varphi}}{l g_{t \varphi}+g_{\varphi \varphi}}
$$

is the angular velocity of the orbiting matter relative to distant observers and

$$
l=-\frac{U_{\varphi}}{U_{t}}
$$

is its specific angular momentum; $U_{t}, U_{\varphi}$ are the covariant components of the 4velocity field of the orbiting matter.

Using (9) we arrive to the formula

$$
\Omega=\frac{\left(1-\frac{2 r-b}{\Sigma}\right) l+\frac{a(2 r-b)}{\Sigma} \sin ^{2} \theta}{\left(r^{2}+a^{2}+\frac{2 r-b}{\Sigma} a^{2} \sin ^{2} \theta\right) \sin ^{2} \theta-l \frac{a(2 r-b)}{\Sigma} \sin ^{2} \theta} .
$$




\section{$\Delta a(b)$}

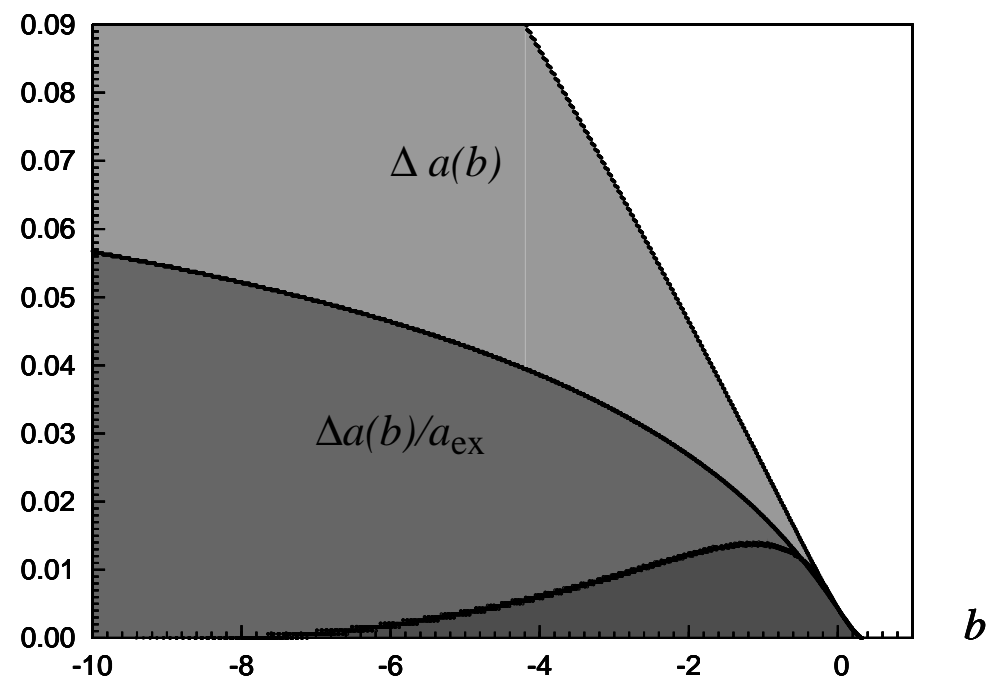

Figure 8. Spin range of braneworld Kerr black holes allowing for the Aschenbach effect given as a function of the tidal charge $b$. It is illustrated by the function $\Delta a(b)$ and the relative spin range determined by the function $\Delta a(b) / a_{\mathrm{ex}}$. The darkest area represents most interesting case (from astrophysical point of view) when the radius of marginally stable orbit $r_{\mathrm{ms}}$ is less than the local minimum of the Keplerian velocity profile.

\section{Velocity profiles for the Keplerian distribution of the specific angular momentum}

\subsection{Circular geodesics}

Motion of test particles following circular geodetical orbits in the equatorial plane $(\theta=\pi / 2)$ is described by the Keplerian distribution of the specific angular momentum, which in the braneworld Kerr backgrounds takes the form [33]

$$
l_{\mathrm{K} \pm}(r ; a, b)= \pm \frac{\left(r^{2}+a^{2}\right) \sqrt{r-b} \mp a(2 r-b)}{r^{2}-2 r+b \pm a \sqrt{r-b}} ;
$$

the signs \pm refer to two distinct families of orbits in the Kerr braneworld spacetimes. From the LNRF point of view the 2nd family, or minus-family (given by the lower sign), represents retrograde orbits, while the 1st family, or plus-family (given by the upper sign), represents direct orbits in the black hole spacetimes [19], but can represent both direct and retrograde orbits in the naked singularity spacetimes. We can find a similiar situation for Kerr spacetime in $[15,16]$. For both families, we find a formal limit of the Keplerian motion to be located at $r=b$. In the black hole spacetimes, it is located under the inner horizon and is irrelevant from the astrophysical point of view. In the naked singularity spacetimes, it is relevant for $b>0$, while for $b<0$, it is located under the ring singularity at $r=0$ which represents the limit on the location of circular orbits. 

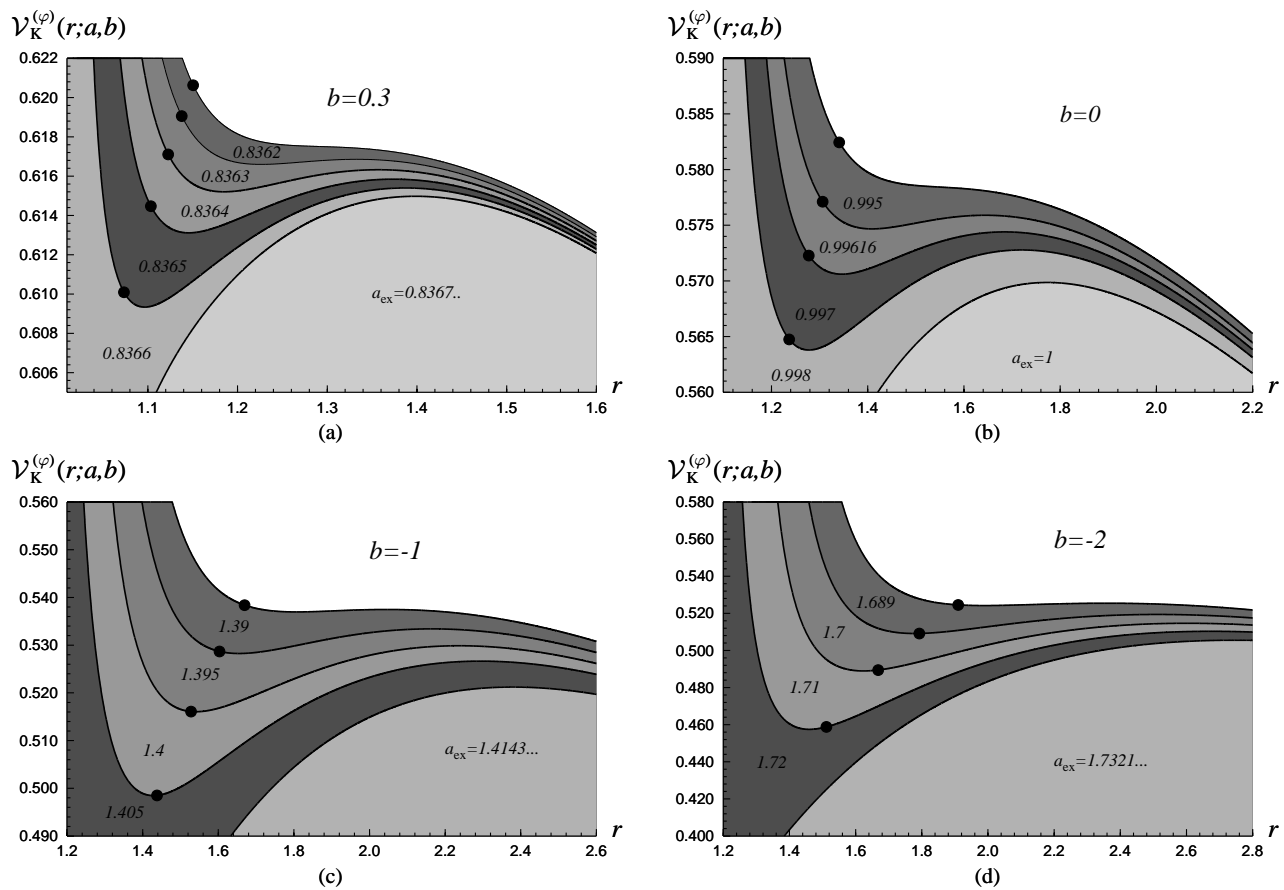

Figure 9. Non-monotonic LNRF-related velocity profiles for braneworld Kerr black hole backgrounds given for some values of the tidal charge $b$ and appropriately chosen values of the $a$. The black points denote loci of $r_{\mathrm{ms}}$.

The corresponding Keplerian angular velocity related to distant observers is given by the relation

$$
\Omega_{\mathrm{K} \pm}(r ; a, b)= \pm \frac{1}{r^{2} / \sqrt{r-b} \pm a},
$$

and the Keplerian orbital velocity related to the LNRF in braneworld Kerr backgrounds is thus given by the relation

$$
\mathcal{V}_{\mathrm{K} \pm}^{(\varphi)}(r ; a, b)= \pm \frac{\sqrt{r-b}\left(r^{2}+a^{2}\right) \mp a(2 r-b)}{\left(r^{2} \pm a \sqrt{r-b}\right) \sqrt{\Delta}} .
$$

Clearly, there is a limit on existence of circular equatorial geodesics at $r=b$ that is relevant (and located above the ring singularity) for spacetimes with positive tidal charge.

Like in the Kerr backgrounds $(b=0)$, also in the braneworld Kerr backgrounds only the plus-family orbits exhibit the "minimum-maximum" humpy structure of the LNRF-related orbital velocity profiles $\mathcal{V}_{\mathrm{K}}^{(\varphi)}(r)$. On the other hand, in braneworld Kerr naked-singularity spacetimes with positive tidal charge $(b>0)$, there is always at least one local extreme in the orbital velocity profiles (maximum in $\mathcal{V}_{\mathrm{K}+}^{(\varphi)}$ and minimum in $\mathcal{V}_{\mathrm{K}-}^{(\varphi)}$ profiles) where the orbital velocity gradient changes its sign. This non-monotonicity, however, does not correspond to the Aschenbach effect.

We illustrate the typical character of $\mathcal{V}_{\mathrm{K}+}^{(\varphi)}(r ; a, b)$ velocity profiles in the braneworld rotating black hole and naked singularity spacetimes with negative tidal charge in Figure 2 - in this case the profiles are extended down to $r=0$, but 
here we do not consider region of $r<0$. For special values of parameters of naked singularity spacetimes, the Aschenbach effect can be related to retrograde region of 1st family orbits (the humpy structure contains orbits with $\mathcal{V}_{K+}^{(\varphi)}<0$ ).

For positive tidal charge the characteristic profiles of $\mathcal{V}_{\mathrm{K}+}^{(\varphi)}$ are presented in the Figure 3 - in this case the profiles finish their validity at $r=b$. There is also depicted velocity profile under the inner horizon of the black hole. Numerical calculations indicate that for those kinds of velocity profiles there is no Aschenbach effect, so for the black hole spacetimes we shall focus our attention on the behaviour of the Keplerian profiles $\mathcal{V}_{\mathrm{K}+}^{(\varphi)}(r ; a, b)$ above the outer horizon.

For both positive and negative tidal chrges the profiles of $\mathcal{V}_{\mathrm{K}-}^{(\varphi)}$ in black hole and naked singularity spacetimes are presented in Figure 4. These profiles do not exhibit any "minimum-maximum" structures. Therefore, in the following we shall focus attention to the plus-family orbits only and use notation $\mathcal{V}_{\mathrm{K}}^{(\varphi)}(r ; a, b)$ instead of $\mathcal{V}_{\mathrm{K}+}^{(\varphi)}(r ; a, b)$.

We have to consider the function $\mathcal{V}_{\mathrm{K}}^{(\varphi)}(r ; a, b)$ (and also the functions $l_{\mathrm{K}}(r ; a, b)$, $\left.\Omega_{\mathrm{K}}(r ; a, b)\right)$ within the range of definition of the Keplerian motion [33]. The range is governed by the radii of the photon circular geodesics $r_{\mathrm{ph}}$ given implicitly by the relation

$$
a=a_{\mathrm{ph}}(r ; b) \equiv \frac{r(3-r)-2 b}{2 \sqrt{r-b}},
$$

and by the radii of the marginally stable circular geodesics $r_{\mathrm{ms}}$, implicitly given by

$$
a=a_{\mathrm{ms}}(r ; b) \equiv \frac{4(r-b)^{3 / 2} \mp r \sqrt{3 r^{2}-2 r(1+2 b)+3 b}}{3 r-4 b} .
$$

The functions $a_{\mathrm{ph}}(r ; b)$ and $a_{\mathrm{ms}}(r ; b)$ are illustrated in Figure 5 - for detailed discussion of the properties of photon and marginally stable orbits see [33,39]. Above the black hole outer horizon, the stable orbits are located in the interval of $r_{\mathrm{ms}}<r<\infty$, while unstable orbits are located in the interval $r_{\mathrm{ph}}<r<r_{\mathrm{ms}}$. Note that for Kerr naked singularity the situation is generally more complicated, see for example [33]. For $r \rightarrow r_{\mathrm{ph}}$ there is $\mathcal{V}_{\mathrm{K}}^{(\varphi)}(r ; a, b) \rightarrow 1$. The stable circular orbits are relevant for Keplerian accretion discs, therefore, it is reasonable to put the limits on the physical relevance of the Aschenbach effect to the region of stable circular geodesics.

It is well known that for standard 4D Kerr black holes the Aschenbach effect, i.e., the non-monotonic LNRF-related orbital velocity profile appears for strongly limited class of near-extreme black holes $[9,21]$. It appears in the regions where the LNRF-related velocity of Keplerian motion reaches relatively large magnitude $\mathcal{V}_{\mathrm{K}}^{(\varphi)} \sim 0.5-0.6$, but the velocity difference of the minimum-maximum hump is much smaller $\left(\Delta \mathcal{V}_{\mathrm{K}}^{(\varphi)} \sim 0.01\right)$. These are the reasons why the effect was overlooked for a relatively long time. On the other hand, the Aschenbach effect is much stronger for Kerr naked singularities and is manifested for a large range of spin $1<a<4.0005$. Moreover, for Kerr naked singularities with spin close to the extreme black hole state $(a=1)$, the Aschenbach effect is connected to another interesting effect related to circular geodesics - namely the retrograde character of the 1st family circular geodesics related to the LNRF. The counter-rotating orbits of the 1st family can constitute a part of the non-monotonic Keplerian profile (see Figure 1 and Figure 


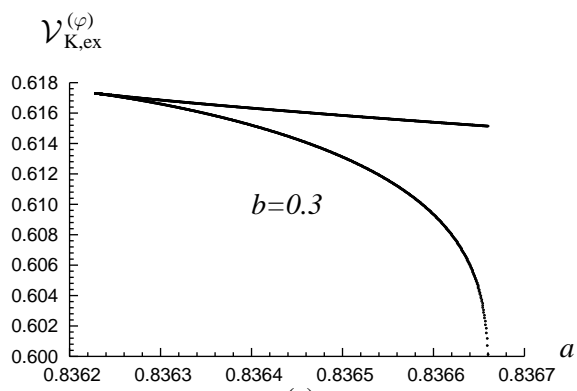

(a)

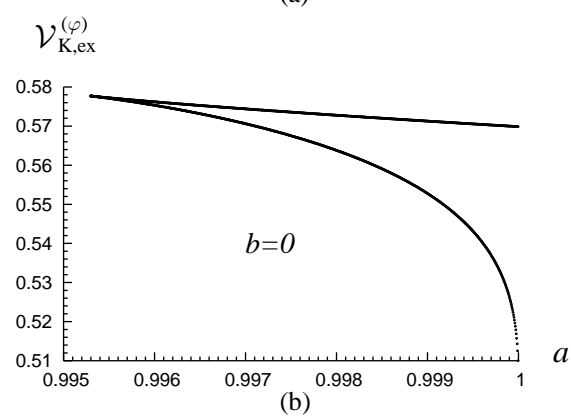

$\mathcal{V}_{\mathrm{K}, \mathrm{ex}}^{(\varphi)}$

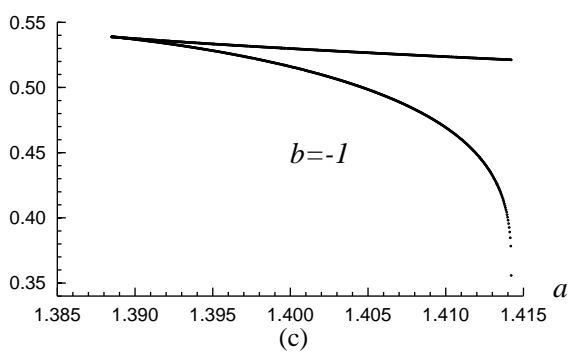

$\mathcal{V}_{\mathrm{K}, \mathrm{ex}}^{(\varphi)}$

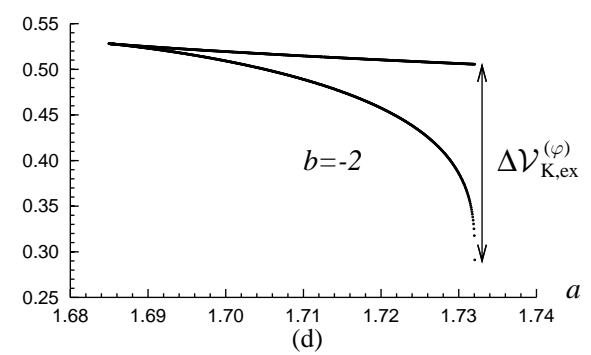

$\mathcal{V}_{\mathrm{K}}^{(\varphi)}\left(r=r_{\mathrm{ms}} ; a, b\right)-\mathcal{V}_{\mathrm{K}}^{(\varphi)}\left(r=r_{\min } ; a, b\right)$

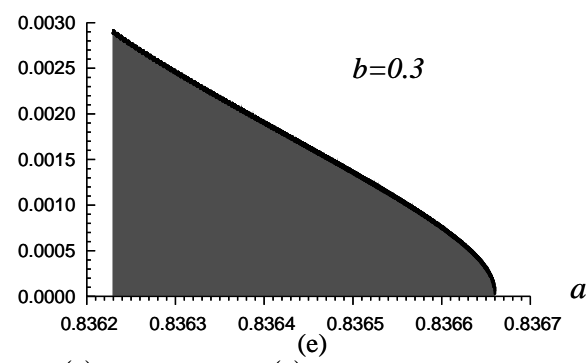

$\mathcal{V}_{\mathrm{K}}^{(\varphi)}\left(r=r_{\mathrm{ms}} ; a, b\right)-\mathcal{V}_{\mathrm{K}}^{(\varphi)}\left(r=r_{\min } ; a, b\right)$

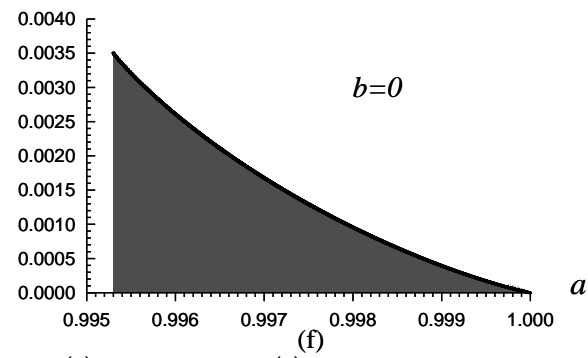

$\mathcal{V}_{\mathrm{K}}^{(\varphi)}\left(r=r_{\mathrm{ms}} ; a, b\right)-\mathcal{V}_{\mathrm{K}}^{(\varphi)}\left(r=r_{\min } ; a, b\right)$

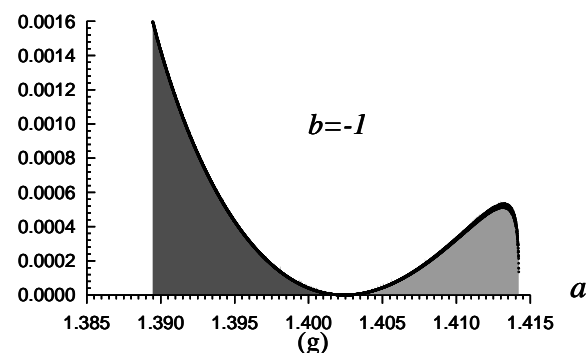

$\mathcal{V}_{\mathrm{K}}^{(\varphi)}\left(r=r_{\mathrm{ms}} ; a, b\right)-\mathcal{V}_{\mathrm{K}}^{(\varphi)}\left(r=r_{\min } ; a, b\right)$

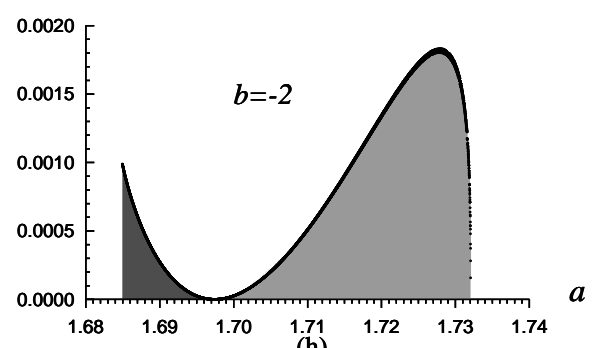

(h)

Figure 10.

(a)-(d) Function $\mathcal{V}_{\mathrm{K} \text {,ex }}^{(\varphi)}$ which determines values of the local minimum and maximum of the function $\mathcal{V}_{\mathrm{K}}^{(\varphi)}$. The upper line represents the local maximum, while the lower line represents the local minimum. These curves demonstrate that maximum of the velocity difference is reached for the extreme black hole states. In (d), there is an indication for the definition of extremal velocity difference (occurring for the extreme black hole states) as a function of the tidal charge $b$, function $\Delta \mathcal{V}_{\mathrm{K}, \mathrm{ex}}^{(\varphi)}$ giving maximal difference between value of the local maximum and local minimum of the function $\mathcal{V}_{\mathrm{K}, \mathrm{ex}}^{(\varphi)}$, considered with fixed $b$, is in Figure 11.

$(\mathrm{e})-(\mathrm{h})$ Function $\mathcal{V}_{\mathrm{K}}^{(\varphi)}\left(r=r_{\mathrm{ms}}, a, b\right)-\mathcal{V}_{\mathrm{K}}^{(\varphi)}\left(r=r_{\text {min }, a, b}\right)$ which defines difference between $\mathcal{V}_{\mathrm{K}}^{(\varphi)}$ with radius for marginally stable orbit and with radius for local minimum. The dark grey region represent more astrophysicaly interesting cases, when radius of marginally stable orbit $r_{\mathrm{ms}}$ is lower than the radius for the local minimum $r_{\min }$, see Figure 8 
2 ). The "retrograde" Kerr naked singularities manifesting (strongly) the Aschenbach effect, can be determined by the relation (implied by the condition $\mathcal{V}_{\mathrm{K}}^{(\varphi)}=0$ )

$$
a=a_{R \pm}(r) \equiv \sqrt{r}(1 \pm \sqrt{1-r}) .
$$

It is illustrated in Figure 5 (the case $b=0$ ) - we see that the retrograde 1st-family orbits exist at radii $0<r<1$, for spin parameters $1<a_{R+}<3 \sqrt{3} / 4$; the Keplerian LNRF-related velocity profile touches $\mathcal{V}_{\mathrm{K}}^{(\varphi)}=0$ at $r=1$ for $a=1$.

For braneworld Kerr naked singularities, the retrograde motion of plus-family circular geodesics appears for spin determined by the condition $a_{R-}<a<a_{R+}$, where

$$
a_{R \pm}(r ; b) \equiv \frac{(2 r-b) \pm \sqrt{\mathcal{D}}}{2 \sqrt{r-b}}
$$

where

$$
\mathcal{D}=b^{2}-4 r(1-r) b+4 r^{2}(1-r) .
$$

Functions $a_{R \pm}(r ; b)$ are illustrated in Figure 5 . The conditions $\mathcal{D} \geq 0$ and $r>b$ put limit on the radii where for given tidal charge $b$ the retrograde plus-family orbits can exist, as illustrated in Figure 6.

\subsection{Aschenbach effect in braneworld spacetimes}

Character of the LNRF-related velocity profile of the Keplerian (equatorial) circular motion is determined by the behaviour of the velocity gradient that can be expressed in the form

$$
\frac{\partial \mathcal{V}_{\mathrm{K}}^{(\varphi)}}{\partial r}=\frac{A_{1}}{A_{2}}-\frac{A_{3} A_{4}}{A_{2}^{2}}
$$

where

$$
\begin{aligned}
& A_{1}=\sqrt{r-b}\left(\frac{r^{2}+a^{2}}{2(r-b)}+2 r\right)-2 a, \\
& A_{2}=\left(a \sqrt{r-b}+r^{2}\right) \sqrt{\Delta}, \\
& A_{3}=\sqrt{r-b}\left(r^{2}+a^{2}\right)-a(2 r-b), \\
& A_{4}=\sqrt{\Delta}\left[\left(\frac{a}{2 \sqrt{r-b}}+2 r\right)+\frac{r-1}{\Delta}\left(a \sqrt{r-b}+r^{2}\right)\right] .
\end{aligned}
$$

Considering the braneworld Kerr black holes, we restrict our attention to the region above the event horizon at $r>r_{+}=1+\sqrt{1-a^{2}-b}$. The rotation (spin) parameter of black hole spacetimes is limited by

$$
a_{\mathrm{ex}}=\sqrt{1-b}
$$

for a given tidal charge $b$. When braneworld Kerr naked singularities are considered, the region $r>b$ has to be studied for the existence of the humpy LNRF-velocity profiles when $b>0$, while for $b<0$ we have to analyse the whole region of $r>0$ above the ring singularity. 
Local extrema of $\mathcal{V}_{\mathrm{K}}^{(\varphi)}(r)$ profiles (giving their "minimum-maximum" humpy parts) are determined by the condition $\partial \mathcal{V}_{\mathrm{K}}^{(\varphi)} / \partial r=0$, i.e., by zero points of the function (31) that are identical to the roots of the polynomial

$$
g(Z): \sqrt{r}\left(h_{1} Z^{4}+h_{2} Z^{3}+h_{3} Z^{2}+h_{4} Z+h_{5}\right)=0,
$$

where

$$
\begin{aligned}
h_{1} & =r\left[3 r^{2}+2(1-2 b) r-3 b\right], \\
h_{2} & =-2 r^{2}(1-b / r)^{3 / 2}(3 r+1), \\
h_{3} & =4 r^{4}-2(5+3 b) r^{3}+16 b r^{2}+2 b r(1-3 b)-b^{2}, \\
h_{4} & =\sqrt{1-b / r}\left\{2 r^{3}\left[4(1-b / r)^{2}+4(1-b / r)+1\right]-\right. \\
& \left.-2 r^{4}[1+4(1-b / r)]\right\}, \\
h_{5} & =r^{3}\left(r^{2}-2 b r+b\right) .
\end{aligned}
$$

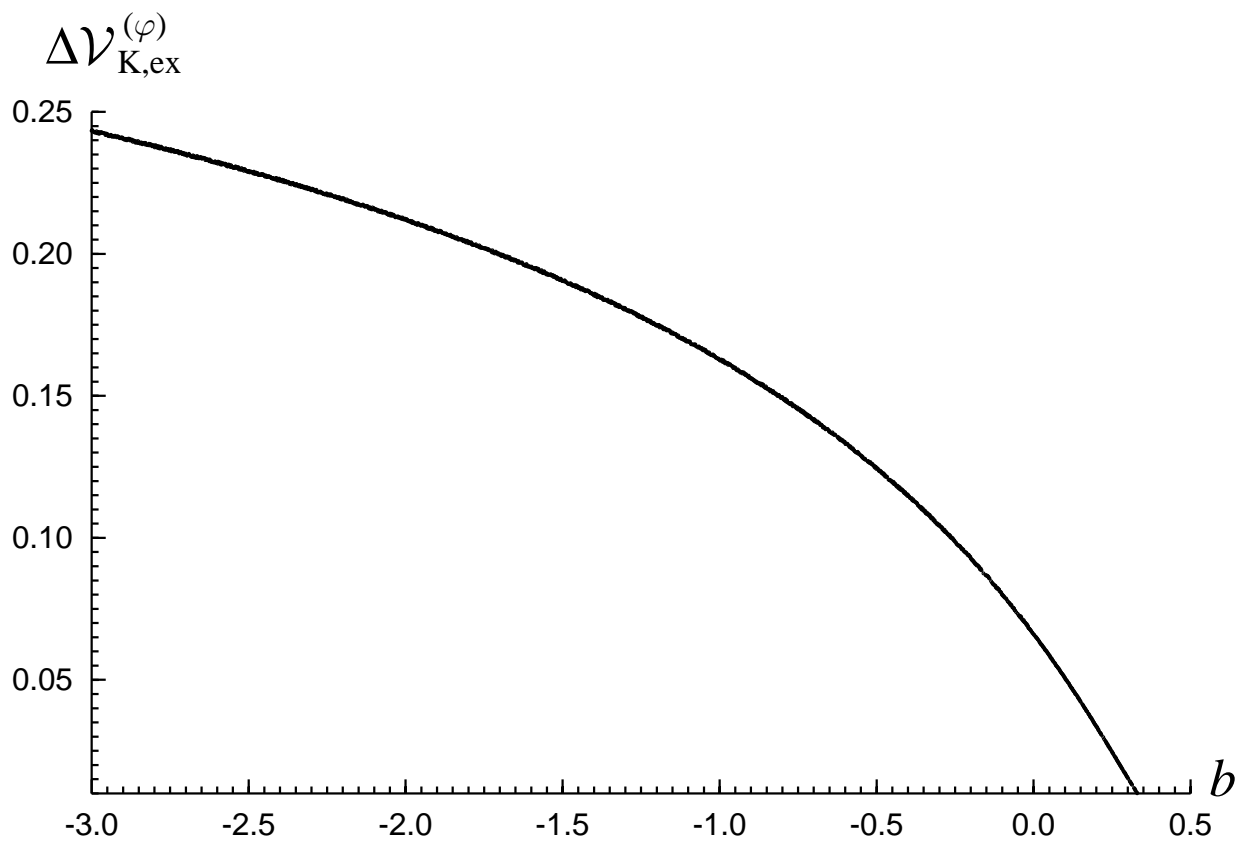

Figure 11. Function $\Delta \mathcal{V}_{\mathrm{K}, \mathrm{ex}}^{(\varphi)}$ giving maximal difference between values of the local maximum and local minimum of the function $\mathcal{V}_{\mathrm{K}, \mathrm{ex}}^{(\varphi)}$, for a fixed $b$.

The occurrence of the Aschenbach effect can be analysed by numerical study of the roots of Eq. (37). The numerical approach enables to determine the region in the parameter space $(a-b)$ where this effect is admitted, see Figure 7. In naked singularity region, there is a subregion with retrograde 1st-family orbits. We can see that for black holes the region is highly sensitive on the choice of the tidal charge $b$. For positive values of $b$ increasing, it becomes narrowed as compared with the case of $b=0$ and disappears when $b>0.41005$. In the limit value of $b=0.41005$, the corresponding 


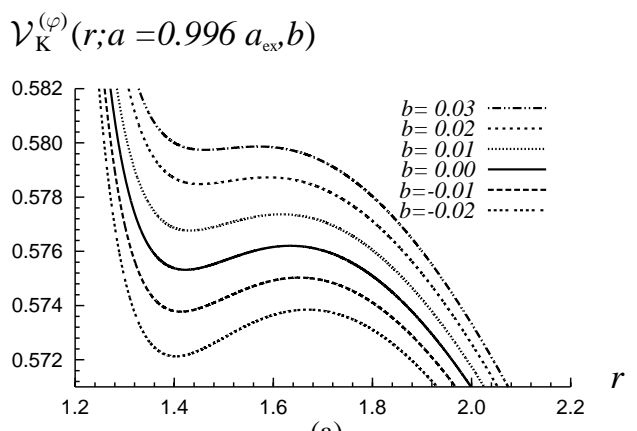

(a)

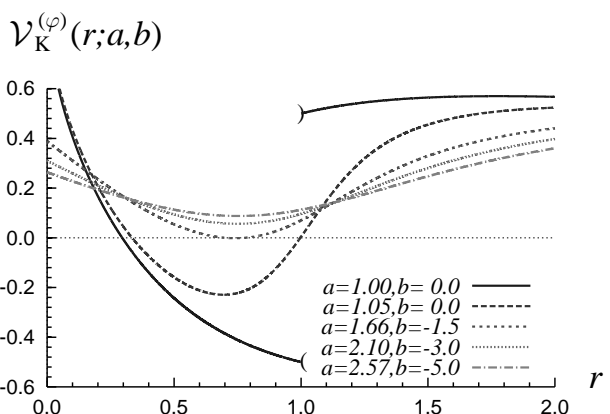

(b)

Figure 12. (a) Sequence of non-monotonic LNRF-related velocity profiles for near extreme black hole background. In the sequence, there is $a=0.996 a_{\mathrm{ex}}$ and we change the parameter $b$.

(b) Sequence of non-monotonic LNRF-related velocity profiles for naked singularity background close to the extreme black hole state given by $a_{\mathrm{ex}}=$ $\sqrt{1-b}$. In the sequence, there is $a=1.05 a_{\mathrm{ex}}$, and we can see the transform between the retrograde and purely corotating velocity profiles. For comparison, the velocity profile constructed for the extreme Kerr black hole is included.

value of the spin reads $a=0.76808$. On the other hand, the interval of the black hole spin allowing for the Aschenbach effect enlarges significantly with descending of negative tidal charge $b$. We demonstrate this dependence in Figure 8 presenting the function $\Delta a(b)$, which denotes spin interval of the black holes (with tidal charge $b$ ) allowing for the Aschenbach effect. Since the maximal black hole spin $a_{\text {ex }}$ also depends on $b$, we give the dependence of the ratio $\Delta a / a_{\mathrm{ex}}$ on $b$ for completeness. Notice that for tidal charge $b=-1$, the spin interval $\Delta a$ (and even its relative magnitude given by $\left.\Delta a / a_{\mathrm{ex}}\right)$ increases by almost one order as compared to the case of $b=0$. In the naked singularity spacetimes, the Aschenbach effect appears in wide region of $a, b$ parameters - both for positive and negative tidal charges. The allowed naked singularity region is limited by $b=2.14$ and $a=5.99$ - the point $Q$ in Figure 8 .

The behaviour of humpy LNRF-velocity profiles of Keplerian orbits in the field of braneworld Kerr black holes is represented by a series of figures for both positive and negative tidal charges. In order to clearly illustrate all the aspects of the Aschenbach effect in dependence on the black hole parameters, we give figures with both fixed value of the tidal charge $b$ and fixed value of the rotation parameter $a$, see Figure 9 . (The boundary of stable orbits given by the marginally stable orbit is represented by a point on all the profiles.) Moreover, we include the dependences of the LNRF velocities in the minimum and maximum of the humpy profile on the black hole parameters (see Figure 10). These extremal LNRF-related velocities decrease with the tidal charge descending, but their difference increases significantly - for fixed $b$, the depth of the humpy profile grows with black holes spin approaching $a_{\mathrm{ex}}=\sqrt{1-b}$. Finally, in Figure 11, there is the dependence of the velocity difference at the minimummaximum part of the humpy profile on the tidal charge $b$ for extreme braneworld Kerr black holes. We can se that for $b=-1$ the extremal difference increases by a factor $\sim 2$ as compared to the case of $b=0$.

We also demonstrate there, how the Aschenbach effect disappears about the point $Q$ for positive tidal charges, and how the LNRF-related Keplerian profiles in the naked singularity spacetime can be reduced into those for the corresponding extreme black 
hole spacetime, when the retrograde naked singularity profile is transformed into a discontinuity occurring at the radius $r=1$, and the negatively valued part under the horizon becomes physically irrelevant. Behaviour of Keplerian velocity profiles in both black hole and naked singularity backgrounds corresponding to near-extreme black hole cases is shown in the Figure 12.

\section{Conclusions}

We have shown that the Aschenbach effect is a typical feature of the circular geodetical motion in the field of both standard and braneworld Kerr naked singularities with a relatively large interval of spins above the extreme black-hole limit. For nakedsingularity spin sufficiently close to the extreme black-hole state, the Aschenbach effect is manifested by the retrograde plus-family circular orbits. For black hole spacetimes, such retrograde orbits can appear under the inner horizon, being thus irrelevant from the astrophysical point of view. In the field of near-extreme rotating black holes, the Aschenbach effect located above the outer black hole horizon can be thus considered as a small remnant of typical naked singularity phenomenon.

We have demonstrated that in the braneworld near-extreme Kerr black hole spacetimes, the non-monotonic LNRF-related orbital velocity profiles of the Keplerian motion are suppressed for positive tidal charges as compared with the standard Kerr black holes, and disappear for the tidal charge $b>0.41005$, while they are strongly enlarged for decreasing negative tidal charge. The spin range of black holes allowing for the Aschenbach effect increases significantly with decreasing tidal charge, strengthening possible relevance of the Aschenbach effect in astrophysical phenomena (see, e.g., [50]). This possibility is further supported by increasing magnitude of the velocity difference in the minimum-maximum part of the LNRF-related Keplerian profile with decreasing negative tidal charge. Recent observations of high-frequency quasiperiodic oscillations observed in the X-ray spectra of some neutron-star binaries $[51,52,53,54]$ that could be used to test braneworld models in the strong-field limits imply negatively-valued dimensionless tidal charges as high as $b=-2$ [39] when the Aschenbach effect could be quite significant and well observed [50].

We conclude that the Aschenbach effect can play a significant role in explaining a variety of physical phenomena (optical effects and related line profiles, explanation of a variety of high-frequency quasi-periodic oscillations observed in some microquasars and active galactic nuclei, etc.) expected to appear in the strong gravitational field of braneworld Kerr black holes, especially in the case of negative tidal charge.

\section{Acknowledgment}

The presented work was supported by the Czech grants MSM 4781305903, LC 06014, GAČR 205/09/H033 and the internal grant SGS/2/2010.

\section{References}

[1] M. Volonteri, P. Madau, E. Quataert, and M. Rees. The Distribution and Cosmic Evolution of Massive Black Hole Spins. Astrophys. J., 620:69-77, 2005.

[2] S. Shapiro. Spin, Accretion, and the Cosmological Growth of Supermassive Black Holes. Astrophys. J., 620:59-68, 2005.

[3] Y. Tanaka, K. Nandra, A. C. Fabian, H. Inoue, C. Otani, T. Dotani, K. Hayashida, K. Iwasawa, T. Kii, H. Kunieda, F. Makino, and M. Matsuoka. Gravitationally Redshifted Emission 
Implying an Accretion Disk and Massive Black-Hole in the Active Galaxy MCG-6-30-15. Nature, 375:659, 1995.

[4] T. Miyakawa, K. Ebisawa, Y. Terashima, F. Tsuchihashi, H. Inoue, and P. Zycki. Spectral Variation of the Seyfert 1 Galaxy MCG 6-30-15 Observed with Suzaku. Publications of the Astronomical Society of Japan, 61:1355, 2009.

[5] C. S. Reynolds, A. C. Fabian, L. W. Brenneman, G. Miniutti, P. Uttley, and L. C. Gallo. Constraints on the absorption-dominated model for the X-ray spectrum of MCG-6-30-15. Monthly Notices Roy. Astronom. Soc., 397:L21-L25, 2009.

[6] J. E. McClintock, R. Shafee, R. Narayan, R. A. Remillard, S. W. Davis, and L.-X. Li. The Spin of the Near-Extreme Kerr Black Hole GRS 1915+105. Astrophys. J., 652:518-539, 2006.

[7] G. Török, M. A. Abramowicz, W. Kluźniak, and Z. Stuchlík. The orbital resonance model for twin peak kHz quasi periodic oscillations in microquasars. Astronomy and Astrophysics, 436:1-8, 2005.

[8] J. F. Steiner, J. F. McClintock, J. A. Orosz, R.Narayan, M. A. Torres, and R. A. Remillard. Estimating The Spin Of The Stellar-Mass Black Hole XTE J1550-564 AAS/High Energy Astrophysics Division, vol 10, 2008.

[9] B. Aschenbach. Measuring mass and angular momentum of black holes with high-frequency quasi-periodic oscillations. Astronomy and Astrophysics, 425:1075-1082, 2004.

[10] G. Török. QPOs in microquasars and Sgr A*: measuring the black hole spin. Astronom. Nachr., 326(9):856-860, 2005.

[11] L. Meyer, A. Eckart, R. Schdel, W. J. Duschl, K. Mużić, M. Doviak, and V. Karas. Near-infrared polarimetry setting constraints on the orbiting spot model for Sgr A* flares. Astronomy and Astrophysics, 460:15, 2006.

[12] E. G. Gimon and P. Hořava. Astrophysical violations of the Kerr bound as a possible signature of string theory. Phys. Lett. B, 672:299-302, 2009.

[13] F. de Felice. Repulsive Phenomena and Energy Emission in the Field of a Naked Singularity. Astronomy and Astrophysics, 34:15, 1974.

[14] F. de Felice. Classical instability of a naked singularity. Nature, 273:429-431, 1978.

[15] Z. Stuchlík. Equatorial circular orbits and the motion of the shell of dust in the field of a rotating naked singularity. Bull. Astronom. Inst. Czechoslovakia, 31(3):129-144, 1980.

[16] Z. Stuchlík. Evolution of Kerr naked singularities. Bull. Astronom. Inst. Czechoslovakia, 32(2):68-72, 1981.

[17] Z. Stuchlík and J. Schee. Appearance of Keplerian discs orbiting Kerr superspinars. Classical and Quantum Gravity, 27(21):215017, 2010.

[18] Z. Stuchlík, S. Hledík, and K. Truparová. Evolution of Kerr superspinars due to accretion counterrotating thin discs. Class. Quantum Grav., 201128155017.

[19] J. M. Bardeen, W. H. Press, and S. A. Teukolsky. Rotating black holes: locally nonrotating frames, energy extraction, and scalar synchrotron radiation. Astrophys. J., 178:347-369, 1972 .

[20] B. Aschenbach. Measurement of Mass and Spin of Black Holes with QPOs. Chinese Journal of Astronomy and Astrophysics Supplement., 8:291-296, 2008.

[21] Z. Stuchlík, P. Slaný, G. Török, and M. A. Abramowicz. Aschenbach effect: Unexpected topology changes in the motion of particles and fluids orbiting rapidly rotating Kerr black holes. Phys. Rev. D (3), 71:024037, 2005.

[22] Z. Stuchlík, P. Slaný, and G. Török. LNRF-velocity hump-induced oscillations of a Keplerian disc orbiting near-extreme Kerr black hole: A possible explanation of high-frequency QPOs in GRS 1915+105. Astronomy and Astrophysics, 470(2):401-404, 2007.

[23] Z. Stuchlík, P. Slaný, and G. Török. Humpy LNRF-velocity profiles in accretion discs orbiting almost extreme Kerr black holes. A possible relation to quasi-periodic oscillations. Astronomy and Astrophysics, 463:807-816, 2007.

[24] P. Slaný and Z. Stuchlík. Mass estimate of the XTE J1650-500 black hole from the extended orbital resonance model for high-frequency QPOs. Astronomy and Astrophysics, 492:319-322, 2008.

[25] Z. Stuchlík, P. Slaný, and G. Török. Humpy LNRF-velocity profiles in accretion discs orbiting rapidly rotating Kerr black holes. In H. Kleinert and R. T. Jantzen, editors, Proceedings of The Eleventh Marcel Grossmann Meeting on Recent Developments in Theoretical and Experimental General Relativity, Gravitation and Relativistic Field Theories, Berlin, Germany, 23-29 July 2006, pages 1060-1062, Singapore, 2008. World Scientific. Series Ed. R. Ruffini.

[26] A. Müller and B. Aschenbach. Non-monotonic orbital velocity profiles around rapidly rotating Kerr-(anti-)de Sitter black holes. Classical Quantum Gravity, 24(10):2637-2644, 2007. 
[27] P. Slaný and Z. Stuchlík. On non-monotonic orbital velocity profiles around rapidly rotating Kerr-(anti)de Sitter black holes: a comment to the recently published results. Classical Quantum Gravity, 25:038001, 2008.

[28] L. Randall and R. Sundrum. A Large Mass Hierarchy from a Small Extra Dimension. Phys. Rev. D, 83(2):3370-3373, 1999.

[29] C. Germani and R. Maartens. Stars in the braneworld. Phys. Rev. D (3), 64:124010, 2001.

[30] P. Figueras and T. Wiseman. Gravity and large black holes in Randall-Sundrum II braneworlds. arXiv:1105.2558, 2011.

[31] A. N. Aliev and A. E. Gümrükçüoğlu. Charged rotating black holes on a 3-brane. Phys. Rev. $D, 71(10): 104027,2005$.

[32] N. Dadhich, R. Maartens, P. Papadopoulos, and V. Rezania. Black holes on the brane. Phys. Lett. B, 487:1-6, 2000

[33] Z. Stuchlík and A. Kotrlová. Orbital resonances in discs around braneworld Kerr black holes. Gen. Relativity Gravitation, 41:1305-1343, 2009.

[34] A. N. Aliev and P. Talazan. Gravitational effects of rotating braneworld black holes. Phys. Rev. D 80 044023, 2009.

[35] J. Schee and Z. Stuchlík. Profiles of emission lines generated by rings orbiting braneworld Kerr black holes. Gen. Relativity Gravitation, 41:1795-1818, 2009.

[36] J. Schee and Z. Stuchlík. Optical Phenomena in the Field of Braneworld Kerr Black Holes. Int. J. Modern Phys. D, 18:983-1024, 2009.

[37] A. Abdujabbarov and B. Ahmedov. Test particle motion around a black hole in a braneworld. Phys. Rev. D (3), 81:044022, 2010.

[38] A. Y. Bin-Nun. Relativistic images in Randall-Sundrum II braneworld lensing. Phys. Rev. D, $81: 123011,2010$.

[39] A. Kotrlová, Z. Stuchlík, and G. Török. Quasiperiodic oscillations in a strong gravitational field around neutron stars testing braneworld models. Classical Quantum Gravity, 25:225016, 2008.

[40] A. I. Mamadjanov, A. A. Hakimov, and S. R. Tojiev. Quantum Interference Effects in Spacetime of Slowly Rotating Compact Objects in Braneworld. Modern Phys. Lett. A, 25:243-256, 2010.

[41] V. S. Morozova, B. J. Ahmedov, A. A. Abdujabbarov, and A. I. Mamadjanov. Plasma magnetosphere of rotating magnetized neutron star in the braneworld. Astronomy and Astrophysics, 330:257-266, 2010

[42] V. S. Morozova and B. J Ahmedov. Electromagnetic Fields of Slowly Rotating Compact Magnetized Stars in Braneworld. Astrophys. and Space Sci., 2010332 133-42

[43] J. Hladík and Z. Stuchlík. Photon and neutrino redshift in the field of braneworld compact stars. Journal of Cosmology and Astroparticle Physics, 2011 JCAP07(2011)012

[44] C. G. Böhmer, T. Harko, and F. S. N. Lobo. Solar system tests of brane world models. Classical Quantum Gravity, 25(4):5015, 2008.

[45] C. G. Böhmer, G. De Risi, T. Harko, and F. S. N. Lobo. Classical tests of general relativity in brane world models. Classical Quantum Gravity, 27:185013, 2010.

[46] CMS Collaboration. Search for Microscopic Black Hole Signatures at the Large Hadron Collider. arXiv:1012.3375v1, 2010.

[47] T. Shiromizu, K.-I. Maeda, and M. Sasaki. The Einstein Equations on the 3-Brane World. Phys. Rev. D, 62:024012, 2000.

[48] B. Carter. Black hole equilibrium states. In C. De Witt and B. S. De Witt, editors, Black Holes, pages 57-214, New York-London-Paris, 1973. Gordon and Breach.

[49] C. W. Misner, K. S. Thorne, and J. A. Wheeler. Gravitation. W. H. Freeman and Co, New York, San Francisco, 1973.

[50] J. Svoboda, M. Dovčiak, R. Goosmann, and V. Karas. Role of emission angular directionality in spin determination of accreting black holes with a broad iron line. Astronomy and Astrophysics, 507:1-17, 2009.

[51] G. Török, M. A. Abramowicz, P. Bakala, M. Bursa, J. Horák, W. Kluzniak, P. Rebusco, and Z. Stuchlík. Distribution of Kilohertz QPO Frequencies and Their Ratios in the Atoll Source $4 \mathrm{U}$ 1636-53. Acta Astronomica, 58:15-21, 2008.

[52] Z. Stuchlík, A. Kotrlová, and G. Török. Black Holes Admitting Strong Resonant Phenomena. Acta Astronomica, $\mathbf{5 8}$ 441, 2008.

[53] G. Török. Reversal of the amplitude difference of $\mathrm{kHz}$ QPOs in six atoll sources. Astronomy and Astrophysics, 497:661-665, 2009.

[54] M. Boutelier, D. Barret, Y. Lin, and G. Török. On the distribution of frequency ratios of $\mathrm{kHz}$ quasi-periodic oscillations. Monthly Notices of the Royal Astronomical Society, 401:1290$1298,2010$. 